\title{
Gene Therapy Approaches for Bone Regeneration
}

\author{
Renny T. Franceschi Shuying Yang R. Bruce Rutherford Paul H. Krebsbach \\ Ming Zhao Dian Wang
}

The Center for Craniofacial Regeneration, University of Michigan School of Dentistry, Ann Arbor, Mich., USA

\section{Key Words}

Adenovirus $\cdot$ Osteoblast $\cdot$ Fracture healing

\begin{abstract}
Gene therapy represents a promising approach for delivering regenerative molecules to specific tissues including bone. Several laboratories have shown that virusbased BMP expression vectors can stimulate osteoblast differentiation and bone formation in vivo. Both in vivo and ex vivo transduction of cells can induce bone formation at ectopic and orthotopic sites. Adenovirus and direct DNA delivery of genes encoding regenerative molecules can heal critical-sized defects of cranial and long bones. Although osteogenic activity can be demonstrated for individual BMP vectors, substantial synergies may be achieved using combinatorial gene therapy to express complimentary osteogenic signals including specific combinations of BMPs or BMPs and transcription factors. Further control of the bone regeneration process may also be achieved through the use of inducible promoters that can be used to control the timing and magnitude of expression for a particular gene. Using these types of approaches, it should be possible to
\end{abstract}

mimic natural processes of bone development and fracture repair and, in so doing, be able to precisely control both the amount and type of bone regenerated.

Copyright @ 2004 S. Karger AG, Basel

\section{Introduction}

Although originally envisioned primarily as a means of correcting genetic defects, gene therapy has recently been used as a means of delivering growth factors, cytokines or morphogens to sites of tissue regeneration. The strategy in this case is to accelerate or induce a natural biological process by expressing a molecule that is normally involved in the regenerative response for the tissue of interest [Bonadio et al., 1999]. This approach is to be contrasted with protein therapy where a recombinant molecule is added directly to the regeneration site. This article will review general concepts concerning the application of gene therapy to bone regeneration including recent work from the author's laboratory. In addition, future novel applications of this therapy will be described that take advantage of the strengths of this technology that allow the simultaneous controlled expression of multiple genes.

\section{KARGER}

Fax +41613061234 E-Mail karger@karger.ch www. karger.com (c) 2004 S. Karger AG, Base

1422-6405/04/1763-0095\$21.00/0

Accessible online at: www. karger.com/cto
Renny T. Franceschi, PhD

Professor of Dentistry and Biological Chemistry

University of Michigan School of Dentistry

1011 N. University Ave., Ann Arbor, MI 48109-1078 (USA)

Tel. +1 743763 7381, Fax +1 743763 5503, E-Mail rennyf@umich.edu 


\section{Gene therapy strategies}

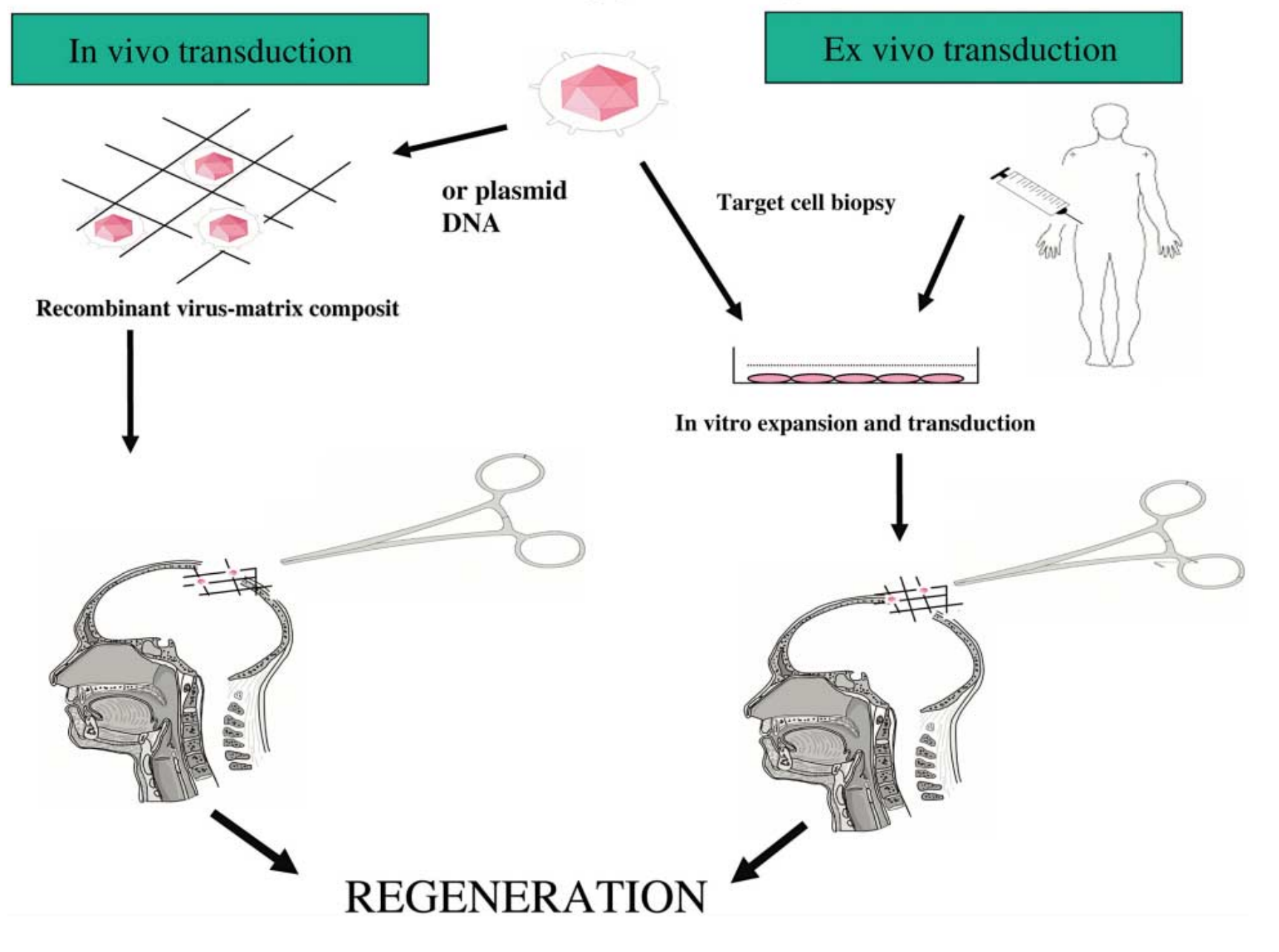

Fig. 1. Strategies for delivering therapeutic genes to tissue sites. In vivo transduction involves direct delivery of a viral or nonviral gene therapy vector to the target tissue of interest using a suitable carrier matrix. Ex vivo gene therapy first requires transduction of syngeneic cells in tissue culture followed by implantation at the regeneration site of the patient.

\section{Methods of Gene Delivery}

In the case of bone, a number of regeneration-associated genes may be considered for gene therapy such as those encoding soluble growth factors (PDGF, FGFs, IGFs), morphogens (BMPs, hedgehog proteins), systemic anabolic factors (PTH, PTHrP, growth hormone), angiogenic factors (VEGF isoforms and FGFs), transcription factors associated with bone/cartilage-related gene expression (Runx2/Cbfa1, Osterix, Sox9, homeodomain proteins such as Dlx2-6, Msx), extracellular matrix molecules associated with induction or inhibition of mineralization (bone sialoprotein, dentin sialophosphoprotein, matrix Gla protein, osteopontin) or receptors/receptor antagonists (soluble IL-1R, soluble BMP receptors). As summarized in figure 1, there are two general ways to deliver a gene to a regenerative site; the gene of choice can be delivered directly to the tissue of interest where it will be taken up by host cells that will then express the regenerative molecule (in vivo transduction) or the gene can be intro- 
duced into cultured cells which are then implanted into the patient (ex vivo transduction). In the later case, implanted cells are normally obtained from the patient to avoid problems with immune rejection of the engrafted cells. Each approach has its own advantages and disadvantages. In the case of in vivo gene therapy, morbidity associated with the harvesting of patient cells for ex vivo transduction are avoided. However, there is much less control over the specific cell population that becomes genetically modified and there can be acute immune responses against the vector particularly in the case of viral vectors where capsid proteins are known to be highly immunogenic [Mahr and Gooding, 1999]. In the case of ex vivo transduction, host cells can be transduced in culture under highly controlled conditions and precisely implanted on a suitable carrier matrix. If the expressed gene encodes a soluble factor, this will then be secreted into the vicinity of the implant where it can affect the transduced cell (autocrine effects) and host cells (exocrine effect). In contrast, if the expressed gene encodes a protein having an intracellular site of action (transcription factors, cell surface receptors, intracellular signal transduction molecules), only the transduced cell will exhibit a direct response to the gene therapy. Such an approach can be used to modify the responsiveness of cells to soluble host factors such as growth factors or hormones.

\section{Gene Delivery Vectors}

Gene delivery vectors can be divided into two general groups, viral and nonviral vectors. Again, each vector type has its own advantages and disadvantages. Because viruses have highly evolved mechanisms to deliver DNA to cells, it is not surprising that they are the most efficient gene delivery vectors known. For example, injection of adenoviral DNA into cells involves cell recognition of specific viral proteins such as the fiber capsid protein by the CAR protein (coxsackievirus and adenovirus receptor) and the viral penton base by $\alpha_{\mathrm{v}}$ integrins [Neumann et al., 1988; Bergelson et al., 1997]. In contrast, nonviral vectors which normally consist of plasmid or related DNA are taken up by cells using a less efficient phagocytotic process. Although recent advances involving condensation of DNA with liposomes or other carriers have the potential to enhance the uptake of nonviral DNA by cells [Kwok et al., 2001], currently the efficiency of this process is approximately $10^{-9}$ that of viral vectors [Franceschi et al., 2000]. In spite of their advantages, viral vectors can be highly immunogenic and, in the case of retroviruses and adeno-associated viruses, can integrate in the host cell genome. Immunogenicity is related to the presence of viral capsid proteins which are known to elicit a potent immune response [Mahr and Gooding, 1999]. Advances in viral vector development have allowed the construction of viral particles in which most of the virus genome has been deleted (so-called gutted vectors) [HartiganO'Connor et al., 1999]. These vectors which require an appropriate cell line to provide the missing capsid proteins are markedly less immunogenic than unmodified viruses. Retroviruses and certain adeno-associated viruses are known to randomly integrate into the host cell genome where they have the potential to disrupt normal gene function by insertional mutagenesis [Hacein-BeyAbina et al., 2003; Noguchi, 2003]. Clearly, major advances in both viral and nonviral vector design will be required to overcome these problems. Nevertheless, for research purposes a number of viral vectors are currently available to efficiently deliver the gene of choice to tissue sites targeted for regeneration.

\section{Biological Activity of an Adenovirus Expressing BMP7}

For the past several years, we have been interested in developing gene therapies for repair and regeneration of mineralized tissues such as craniofacial bones, long bones and teeth. In the following paragraphs, I will describe some of our recent work using adenovirus vectors. These studies were conducted by the author's laboratory in collaboration with other members of the Center for Craniofacial Regeneration at the University of Michigan.

All our reported studies used adenovirus vectors containing the constitutively active cytomegalovirus promoter to drive transgene expression. These vectors were constructed by Cre/lox recombination [Hardy et al., 1997]. Briefly, this method involves cloning the full-length cDNA of interest into a shuttle plasmid (pAdlox) that contains a $5^{\prime}$ viral DNA packaging sequence and a lox $\mathrm{P}$ site adjacent to the expression cassette. Shuttle plasmid and a modified adenovirus genome (Ad5 serotype) also containing lox $\mathrm{P}$ sites flanking the $5^{\prime}$ DNA packaging sequence are transfected into a cell line containing Cre recombinase, thereby allowing recombination between the shuttle plasmid and viral genome. Positive selection for recombinant adenovirus is provided by the presence of the viral DNA packaging sequence adjacent to the expression cassette. 
Table 1. In vitro viral transduction

\begin{tabular}{|c|c|c|c|c|c|}
\hline \multirow{2}{*}{$\begin{array}{l}\text { Viral titer } \\
\text { m.o.i }\end{array}$} & \multicolumn{5}{|c|}{ AdRSVnt-LacZ-positive cells, $\%$} \\
\hline & $\begin{array}{l}\text { MC3T3E1 } \\
\text { osteoblasts }\end{array}$ & $\begin{array}{l}\text { human gingival } \\
\text { fibroblasts }\end{array}$ & $\begin{array}{l}\text { human pulp } \\
\text { fibroblasts }\end{array}$ & $\begin{array}{l}\mathrm{C} 2 \mathrm{C} 12 \\
\text { myoblasts }\end{array}$ & $\begin{array}{l}\text { human } \\
\text { MSCs }\end{array}$ \\
\hline 0 & 0 & 0 & 0 & 0 & 0 \\
\hline 125 & $35 \pm 2$ & $44 \pm 4$ & $92 \pm 5$ & $38 \pm 3$ & $24 \pm 2$ \\
\hline 250 & $63 \pm 5$ & $82 \pm 7$ & $99 \pm 2$ & $79 \pm 2$ & $75 \pm 5$ \\
\hline 500 & $84 \pm 8$ & $98 \pm 2$ & $100 \pm 0$ & $92 \pm 4$ & $90 \pm 5$ \\
\hline 1,000 & $96 \pm 2$ & $100 \pm 0$ & $100 \pm 0$ & $98 \pm 2$ & $100 \pm 5$ \\
\hline
\end{tabular}

Using this method, the author's laboratory developed adenovirus vectors capable of expressing a number of regenerative molecules including BMPs 2, 4 and 7, FGF2 and Runx2. Control adenovirus encoding the bacterial lacZ gene was used to assess the ability of this virus to infect several different cell types. As shown in table 1, this vector can transduce a number of cells relevant to craniofacial regeneration including osteoblasts, gingival and skin fibroblasts and marrow stromal cells. $80-100 \%$ of cells were transduced by virus at a multiplicity of infection (m.o.i. refers to virus particles added/cell) of 500 or less.

Our initial studies on bone induction were conducted using AdCMV-BMP7, an adenovirus engineered to express BMP7 [Franceschi et al., 2000]. In vitro studies demonstrated that cells transduced with this virus produced high concentrations of immunoreactive BMP7. Furthermore, the BMP7 produced was biologically active in that it could induce the transdifferentiation of $\mathrm{C} 2 \mathrm{C} 12$ myoblast cells into osteoblasts, a standard assay for BMP activity [Katagiri et al., 1994]. As described below, both in vivo and ex vivo approaches were used to demonstrate that AdCMV-BMP7 was able to induce bone formation in vivo.

For in vivo transduction, virus was adsorbed to a matrix of BMP-free guanidine-extracted, demineralized bone powder and implanted into subcutaneous or intramuscular sites in CD-1 mice. Control implants contained the same titer of lacZ control virus. Implants were removed for histological analysis after 4 weeks. AdCMVBMP7 formed bone at both subcutaneous and intramuscular sites. Ossicles had a well-defined perimeter of cortical bone with trabeculae and an obvious marrow cavity containing hematopoietic cells as well as fatty marrow surrounding residual carrier. Mineralized areas contained osteocyte-like cells within a bone-like extracellular matrix.
We also used ex vivo transduction to induce bone formation at both soft tissue and orthotopic sites in vivo. The general approach for these studies was to transduce fibroblast or mesenchymal cells with adenovirus in tissue culture, suspend cells in a suitable carrier matrix and implant them into animals. Our published work demonstrates the utility of this approach to induce bone formation at ectopic and orthotopic sites using both gingival or skin fibroblasts [Krebsbach et al., 2000; Rutherford et al., 2002] (also see below). Figures 2 and 3 show more recent studies in which a standardized system was developed for comparing the biological activity of different regenerative molecules using the ectopic bone formation assay. For this work, we used a clonal fibroblast cell line derived from C57BL6 mice (BLK cells) for ex vivo viral transduction. Since C57BL6 mice are syngeneic, they can be implanted with BLK cells without eliciting an immune response. After transduction with AdCMV-BMP7, BLK cells were suspended in a type I collagen hydrogel and subcutaneously implanted. The implanted BLK cells induced ossicles of similar morphology to that seen with in vivo transduction. Ossicles developed via an endochondral process (fig. 2). At early times (3 days), implants contained mainly fibrous tissue. After 1 week, both cartilage and bone were present while at later times (2-4 weeks), cortical and trabecular bone were seen as well as a clearly defined marrow cavity. The amount of bone formed was proportional to the number of BLK cells implanted, increased with time for the first 4 weeks after implantation and persisted for at least 8 weeks (fig. 3a, b). Dose/ response studies revealed that transduction of cells with virus at an m.o.i. of 50-100 was sufficient to maximally induce bone formation with somewhat less bone being formed at higher virus titers. Similarly, the amount of BMP7 secreted by BLK cells in vitro was found to peak over the same range of viral titers (fig. 3c, d). Our earlier work suggested that the bone formed in vivo by AdCMV- 


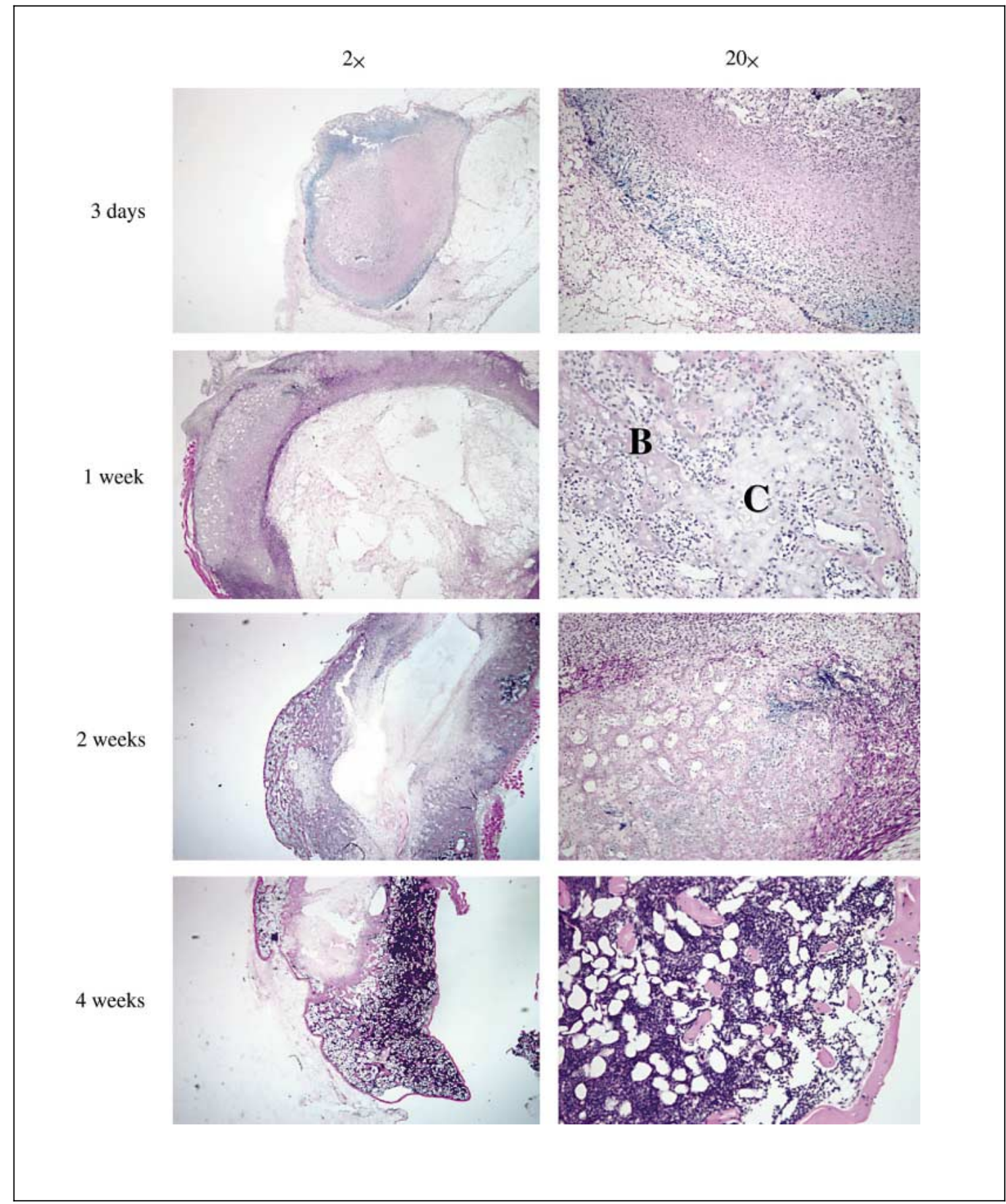

Fig. 2. Induction of endochondral bone formation by BLK cells transduced ex vivo with AdCMV-BMP7; histological analysis. BLK cell cultures were transduced with AdCMV-BMP7 (m.o.i. 100). After $24 \mathrm{~h}$, cells were trypsinized and resuspended in type I collagen hydrogels that were subcutaneously implanted in C57BL/6 mice. At the times indicated, implants were harvested, fixed in $4 \%$ paraformaldehyde, demineralized in $10 \%$ formic acid, dehydrated and embedded in paraffin. Sections $(8 \mu \mathrm{m})$ were cut and stained with hematoxylin and eosin. Bone (B) and cartilage (C) areas are indicated. An ossicle containing trabecular bone, cortical bone and marrow was formed over a 4-week period. 


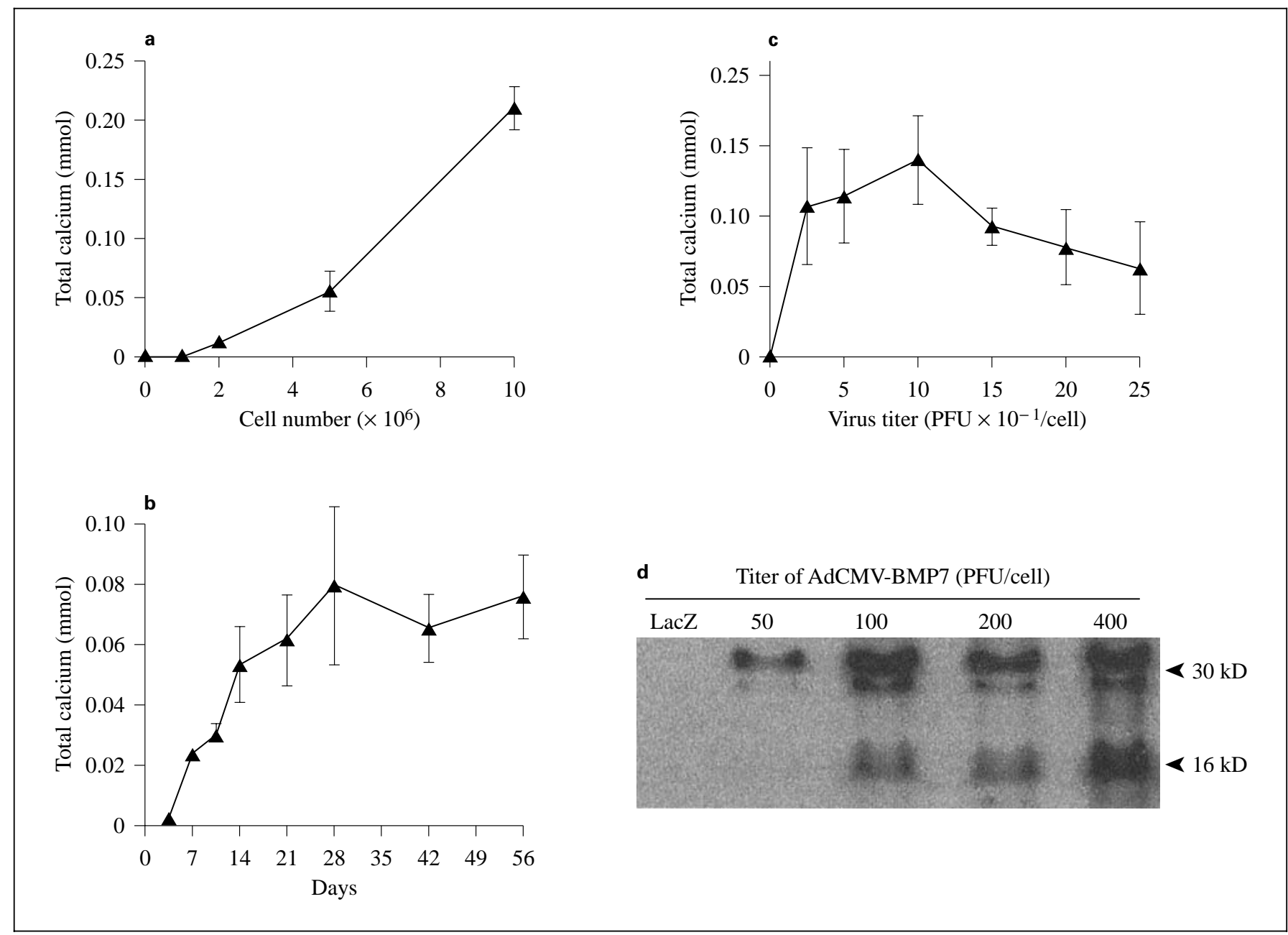

Fig. 3. Quantitation of the osteogenic activity. BLK cells were transduced with AdCMV-BMP7 and implanted into $\mathrm{C} 57 \mathrm{BL} / 6$ mice as described in figure 2. Osteogenesis by implants was assessed by homogenizing ossicles in $15 \%$ trichloroacetic acid and measuring total calcium in supernatants. a Effect of cell number. BLK cells were transduced at an m.o.i. of 100 and the indicated numbers of cells were implanted. Osteogenesis was assessed after 3 weeks. b Time course. BLK cells $\left(1 \times 10^{7}\right.$ cells/implant $)$ were transduced at an m.o.i. of 100 , implanted and harvested at the times indicated. c, d Effect of virus titer. BLK cells were transduced with the indicated virus titers. One set of cells was implanted and harvested after 3 weeks for measurement of bone formation (c) and a second set was incubated in serum-free medium for $24 \mathrm{~h}$ for measurement of BMP7 secretion by Western blotting (d).

BMP7 transduced gingival fibroblasts was composed of host as well as engrafted cells [Krebsbach et al., 2000]. In order to examine the contribution of the implanted BLK cells to ectopic bone, we stably transfected cells with a constitutively active bacterial lacZ gene, transduced them with AdCMV-BMP7 and used these cells in the ectopic bone formation assay. As shown in figure 4, lacZ-marked cells can be detected in implants for up to 6 weeks. Control implants (cells transduced with empty adenovirus) contained lacZ-positive cells in a clearly defined region at the periphery of implanted collagen gels. In AdCMVBMP7-transduced cells, lacZ staining was also initially detected in this region, but at later times the marked cells gradually dispersed throughout the implant such that they represented only about $1-5 \%$ of the total cells in the ossicle. Although osteoblasts were occasionally lacZ positive, most cells were unstained and, therefore, host-derived. Based on these results, we conclude that the predominant 
3 days

2x Control

2x BMP7

20x BMP7
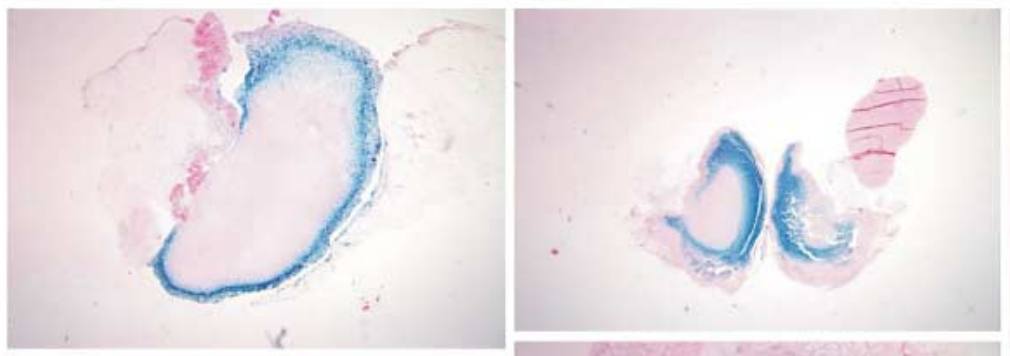

2 weeks
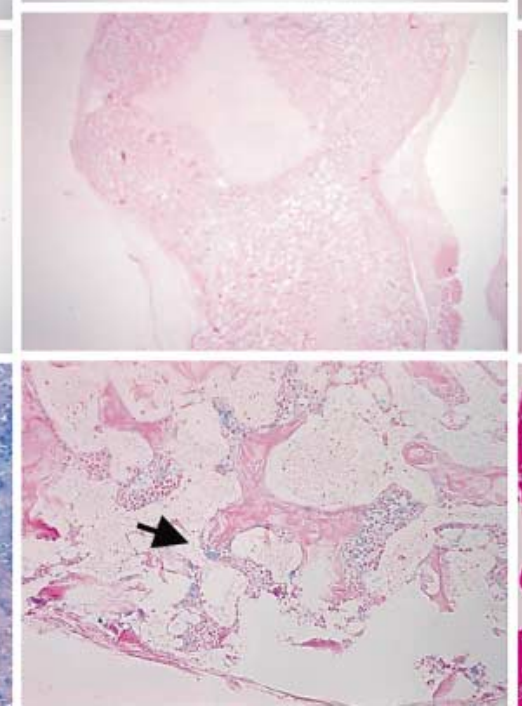

6 weeks

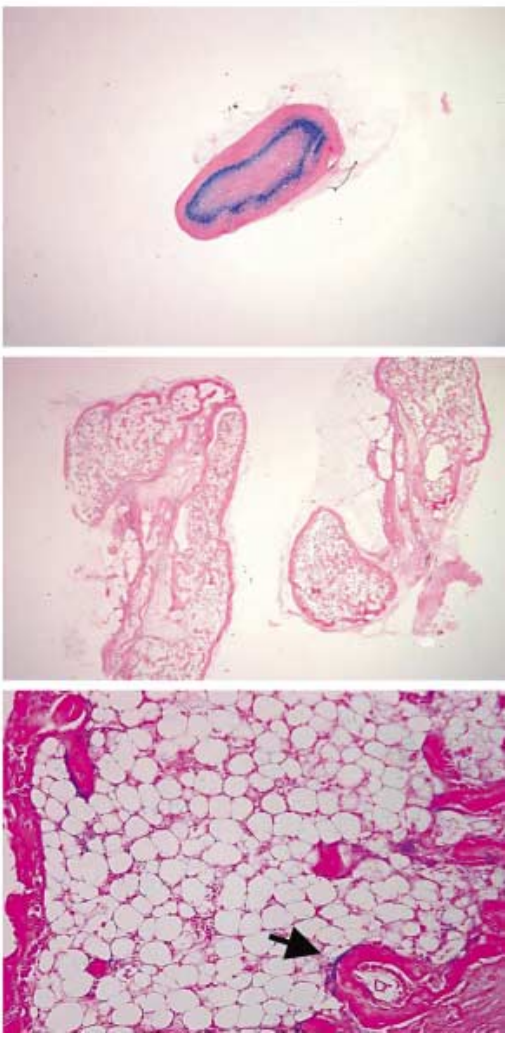

Fig. 4. Retention of implanted BLK (lacZ)-AdCMV-BMP7-transduced cells during bone induction. BLK cells were stably transfected with pCMV-neo-lacZ plasmid encoding the bacterial $\beta$-galactosidase gene. Cells were transduced with empty adenovirus (control) or AdCMV-BMP7 (BMP7), implanted and harvested at the times indicated. Implants were stained for $\beta$-galactosidase activity before paraffin embedding and sectioning. Samples were stained with eosin only to allow visualization of blue $\beta$-galactosidase staining. Blue staining cells associated with osteoblast layers in 2- and 6-week samples are indicated by arrows. Only $2 \times$ magnifications of control implants are shown while $2 \times$ and $20 \times$ magnifications are shown for the AdCMV-BMP7 group. Bone formed by implanted, virally transduced cells was composed primarily of cells from the host.

osteogenic process in this system is the recruitment of host cells by BMP secreted by the genetically engineered BLK cells. Furthermore, the BLK cell/C57BL6 mouse syngeneic system provides a useful means of comparing the osteogenic activity of viruses expressing different regenerative molecules or growth factors.

\section{Use of AdCMV-BMP7 for Regeneration of Cranial and Long Bone Defects}

In studies of greater therapeutic significance, we examined the biological activity of AdCMV-BMP7-transduced fibroblasts in orthotopic regeneration models involving critical sized defects of calvaria and long bones [Krebsbach et al., 2000; Rutherford et al., 2002]. In both cases, studies used syngeneic dermal fibroblasts cultured from Lewis or Fisher rats.

For the cranial model, a 9-mm calvarial defect was created in Lewis rats using a trephine. This size defect will not spontaneously heal in the lifetime of the animal. Cells transduced with either lacZ control adenovirus or AdCMV-BMP7 were adsorbed to gelatin sponges before placement in defects. The AdCMV-BMP7 transduced cells induced sufficient bone formation in 4 weeks to largely close the calvarial defect. In contrast, only minimal bone formation was detected in defects receiving control virus (fig. 5). 


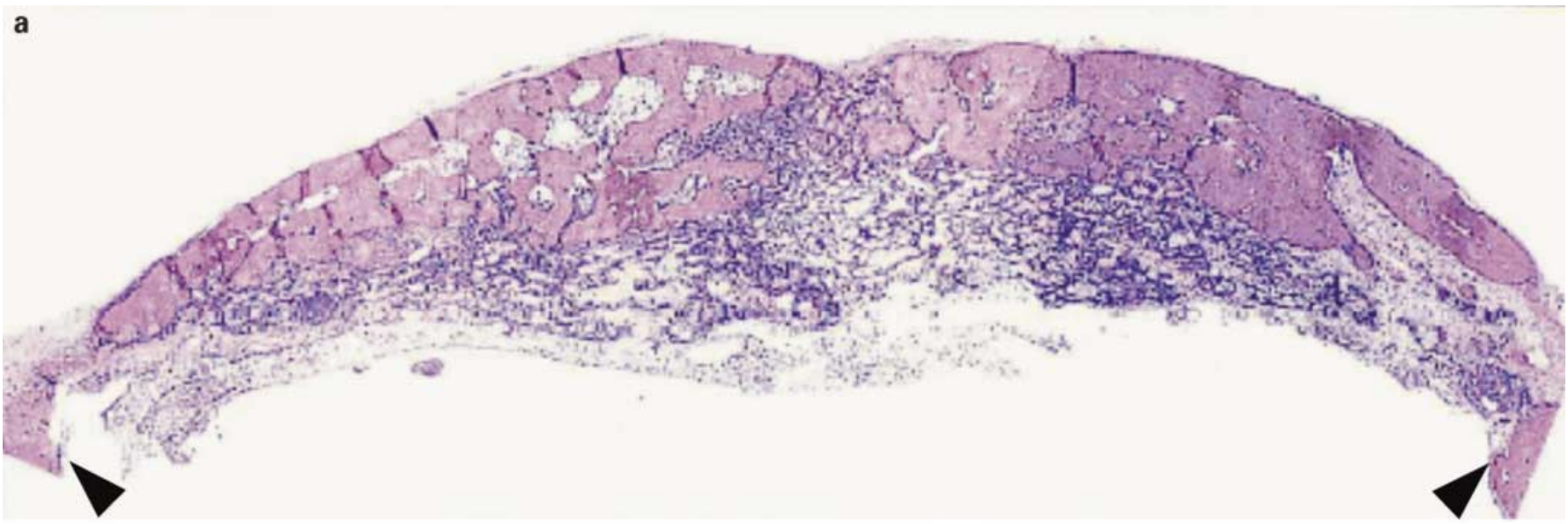

b

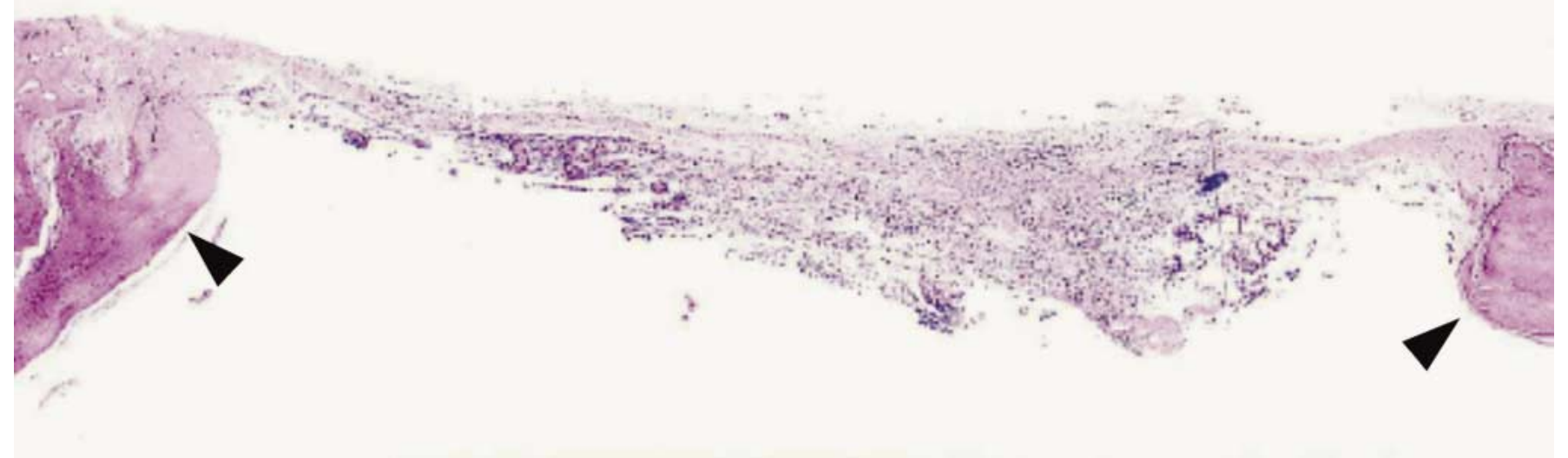

Fig. 5. Regeneration of a critical size cranial defect with AdCMV-BMP7-transduced fibroblasts [from Krebsbach et al., 2000]. Dermal fibroblasts isolated from Lewis rats were transduced with AdCMV-BMP7 (a) or lacZ control virus (b). Cells were adsorbed to a gelatin sponge and placed in a 9-mm-diameter cranial defect created in a Lewis rat host. Calvariae were isolated for histological analysis after 4 weeks. Black arrows designate the surgical margins. Original magnification: $\times 25$. AdCMV-BMP7-transduced cells were able to repair a calvarial defect while control cells produced only fibrous connective tissue.

For the long-bone model, a 2- to 3-mm osteotomy was created in femurs of Fisher rats previously immobilized using an external fixator. Transduced dermal fibroblasts were suspended in a type I collagen hydrogel and adsorbed to a gelatin sponge before placement in the defect. Histological and radiological examination of defects revealed significant bone healing by AdCMV-BMP7-transduced cells after 6 weeks (fig. 6b, d, f). Both bone and cartilage formation was seen. In contrast, lacZ-transduced cells formed only fibrous connective tissue (fig. 6a, c, e).

These studies demonstrate the power of gene therapy to induce bone formation in two clinically relevant models of bone regeneration. Together with studies from other research groups using adenovirus, retrovirus and nonviral vectors [Mason et al., 1998; Lieberman et al., 1999; Baltzer et al., 2000; Okubo et al., 2000; Laurencin et al., 2001; Riew et al., 2003], they provide strong evidence for the feasibility of this approach as a clinical alternative to BMP protein therapy. In related studies, one of us (RBR) showed that AdCMV-BMP7 transduction of tooth pulp can stimulate regeneration of a dentin-like mineralized matrix in vivo [Rutherford, 2001]. This result suggests that BMP gene therapy may also be considered as an alternative to endodontic treatment. 

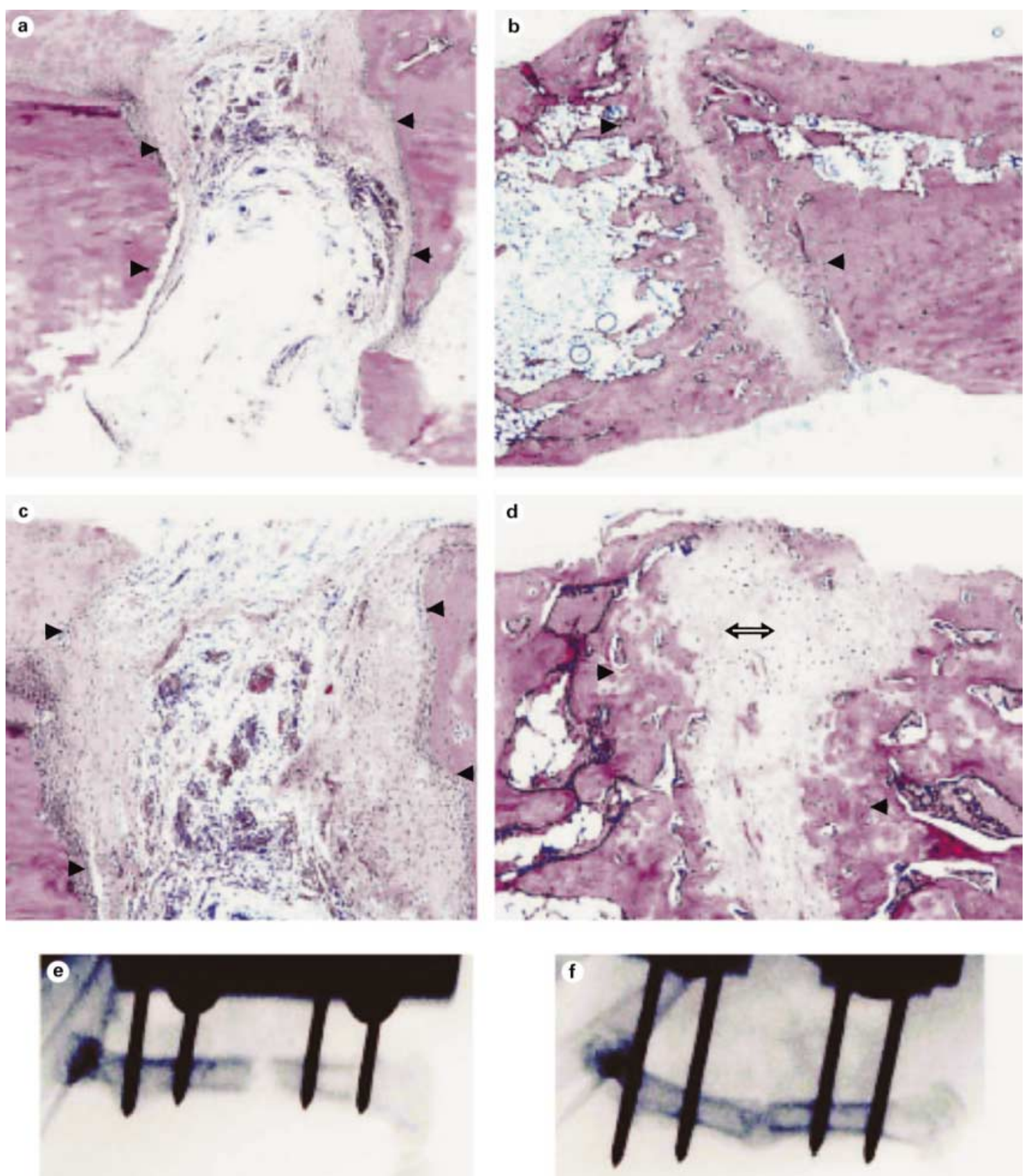

Fig. 6. Regeneration of a long bone critical size defect with AdCMV-BMP7-transduced syngeneic fibroblasts [from Rutherford et al., 2002]. Dermal fibroblasts isolated from Fischer rats were transduced with lacZ control virus (a, c, e) or AdCMV-BMP7 (b, d, f). Cells were suspended in collagen hydrogel, adsorbed to gelatin sponges and placed in 3-mm surgical osteotomies in the femurs of Fischer rats. After 6 weeks, radiographs were taken (e, $\mathbf{f})$ and tissues were processed for histology (a-d). Arrowheads mark margins of the defect. Double arrow identifies cartilage. Original magnification: $10 \times(\mathbf{a}, \mathbf{b})$ and $50 \times(\mathbf{c}, \mathbf{d})$. Partial healing of the long bone defect was only seen with AdCMVBMP7-transduced cells. 

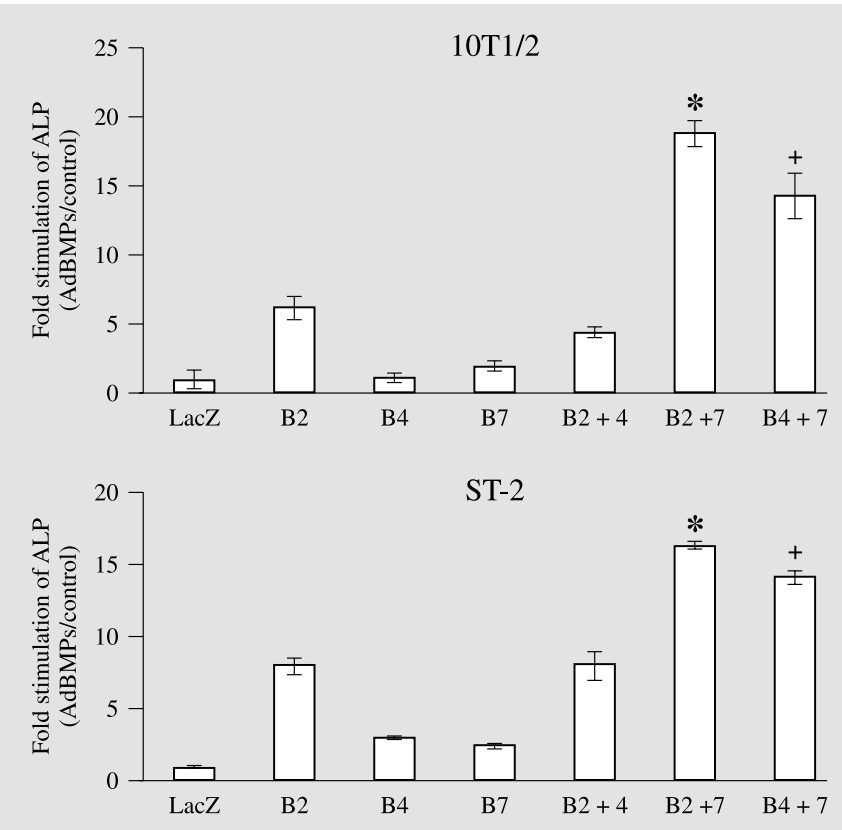

Fig. 7. Cotransduction of mesenchymal cell lines with adenoviruses expressing combinations of BMPs synergistically stimulates alkaline phosphatase activity. C3H10T1/2 and ST2 cells were transduced with the indicated adenoviruses. Cells were harvested after 8 days for measurement of alkaline phosphatase activity and DNA (for normalization). Cotransduction of cells with combinations of either AdCMV-BMP2 or AdCMV-BMP4 with AdCMV-BMP7 stimulated alkaline phosphatase 2-4 times more than would be predicted if effects of individual adenoviruses were additive. 10T1/2= C3H10T1/2 mouse mesenchymal cell line; LacZ = AdCMV-lacZ; B2 = AdCMV-BMP2; B4 = AdCMV-BMP4; B7 = AdCMV-BMP7. Significantly different from the sum of activity in cells individually transduced with AdCMV-BMP2 and AdCMV-BMP7, * $\mathrm{p}<0.001$; significantly different from the sum of activity in cells individually transduced with AdCMV-BMP4 and AdCMV-BMP7, ${ }^{+} \mathrm{p}<0.001$.

\section{Exploiting the Potential of Gene Therapy to Express Combinations of Interacting Genes}

Although the experimental BMP gene therapies described above may eventually lead to therapeutics, they do not exploit the full potential of this approach. Specifically, studies conducted to date involve subcloning of single BMP cDNAs into an appropriate vector which, once transfected into a cell, will direct constitutive BMP production. This approach has several limitations. (1) Because only a single factor is expressed at a time, it fails to adequately mimic normal processes of bone development and fracture healing where multiple secreted factors function in a cooperative and/or sequential fashion to regulate the osteogenic response [Khan et al., 2000; Tickle, 2002]. (2) It is essentially uncontrolled, being limited only by the in vivo half-life of the vector and target cells at the implantation site. (3) It is focused exclusively on the use of soluble factors, yet the osteogenic activity of bone progenitors can also be enhanced by the induction of intracellular components such as transcription factors [Ducy et al., 1997; Yagi et al., 2003]. In view of these limitations, we recently began developing combinatorial gene therapy approaches for bone regeneration that incorporate constitutive or regulated expression of groups of regenerationassociated soluble factors and transcriptional regulators. The remainder of this article will focus on our work in this area. Two examples will be described; the first examines the osteogenic activity of combinations of BMPs and the second shows how the osteogenic activity and BMP responsiveness of an osteoprogenitor population can be enhanced by transducing cells with a virus expressing a bone-related transcription factor.

\section{Synergistic Interactions between BMPs in Stimulating Osteoblast Differentiation}

Although homodimers of BMPs 2, 4 and 7 can induce ectopic bone formation, there is strong evidence that these factors act in combination. For example, BMPs 2, 4 and 7 are coexpressed during development and fracture healing and may exist as heterodimers [Lyons et al., 1995; Khan et al., 2000]. Furthermore, BMP 2/7 and 4/7 heterodimers have greater biological activity than homodimers when assayed for the ability to induce ventral mesoderm and blood in Xenopus animal caps [Nishimatsu and Thomsen, 1998].

Taken together, these studies suggest that certain advantages may be accrued by using combinations of BMPs for bone regeneration. To begin addressing this possibility, we examined the ability of combinations of adenoviruses expressing BMPs 2, 4 and 7 to induce in vitro osteoblast differentiation in two osteoprogenitor cell lines, C3H10T1/2 and ST2 (fig. 7). C3H10T1/2 cells are a pluripotential, mesenchymal cell line derived from mouse embryos [Taylor and Jones, 1979] while ST2 cells are a marrow stromal cell line [Otsuka et al., 1999]. Cells were treated with control (lacZ) virus or with optimal titers of AdCMV-BMP2, 4 or 7 in the combinations indicated. After 8 days, osteoblast differentiation was assessed by measuring alkaline phosphatase activity. When the activity of individual adenoviruses was examined in each cell line, AdCMV-BMP2 was found to have modest activity 


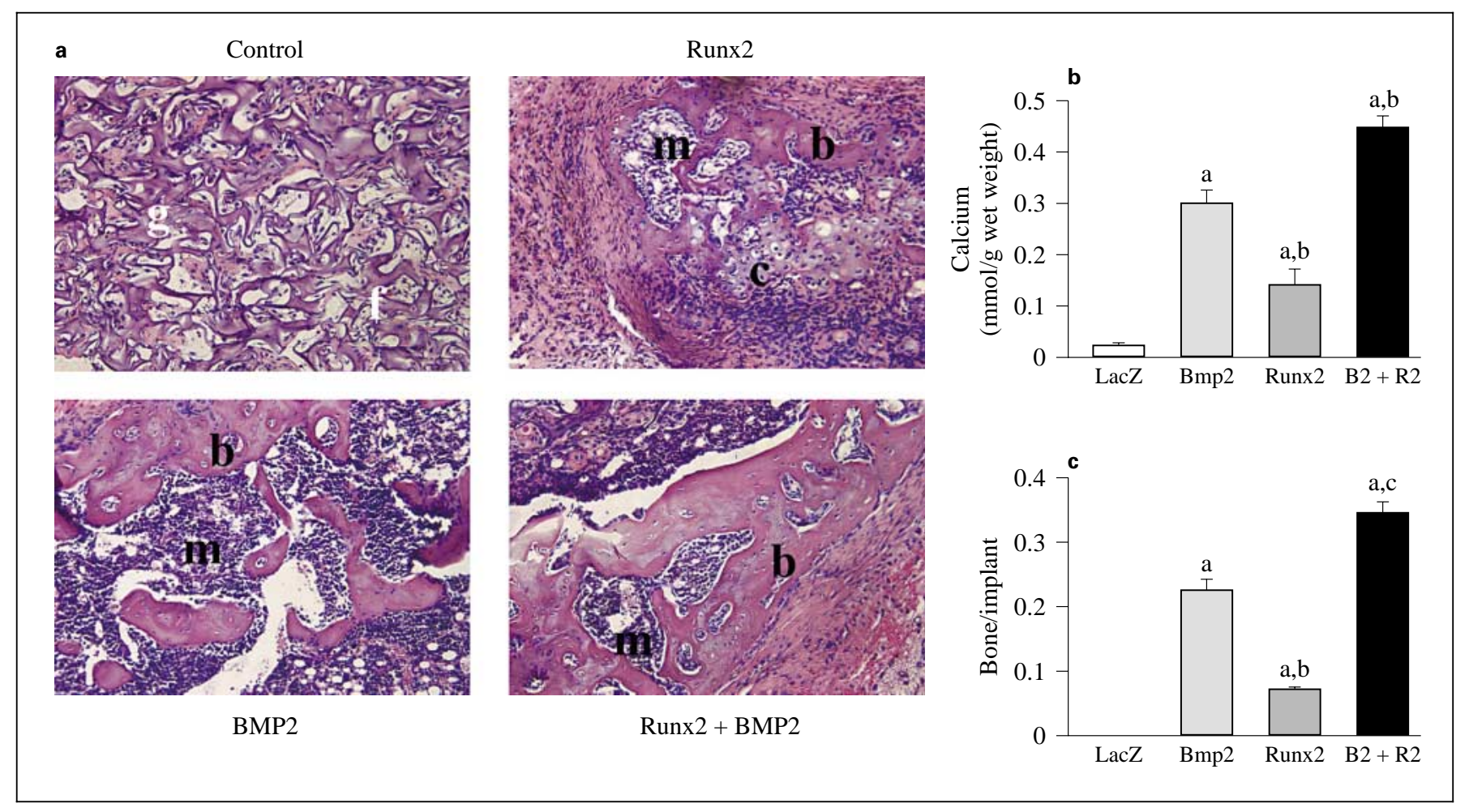

Fig. 8. In vivo bone formation by $\mathrm{C} 3 \mathrm{H} 10 \mathrm{~T} 1 / 2$ cells transduced with adenoviruses expressing Runx2 and BMP2 [from Yang et al., 2003]. Cells were transduced with indicated adenoviruses and implanted into immunodeficient mice. After 4 weeks, transplants were harvested for histological examination (a), determination of total calcium (b) and morphometric analysis (c). For morphometric analysis, results are expressed as the ratio of total bone area/total implant area. Statistical analysis: significantly different from AdLacZ, ${ }^{\mathrm{a}} \mathrm{p}<0.001$; significantly different from AdBmp $2,{ }^{b} p<0.05$. Implants of cells transduced with control virus contained residual gelfoam carrier $(\mathrm{g})$ and fibrous tissue (f), but no bone or cartilage. Cells transduced with AdCMV-Runx2 alone contained small areas of both bone (b) and cartilage (c) as well as a small marrow cavity (m). Both AdCMVBMP2 alone and AdCMV-BMP2 plus AdCMV-Runx2-treated groups formed large ossicles with clearly defined cortical and trabecular bone as well as a marrow cavity. The total amount of bone as measured either by total calcium or fractional bone area was greatest in the AdCMV-BMP2 plus AdCMV-Runx2-treated group. Control = AdCMV-lacZ control virus; $\mathrm{B} 2+\mathrm{R} 2=$ cells transduced with AdCMV-BMP2 plus AdCMV-Runx2. while AdCMV-BMP4 and AdCMV-BMP7 were only slightly more active than the lacZ control virus. Also, when AdCMV-BMP2 and AdCMV-BMP4 were combined, activity was no greater than that seen with AdCMV-BMP2 alone. In contrast, combining AdCMVBMP2 or AdCMV-BMP4 with AdCMV-BMP7 synergistically stimulated alkaline phosphatase activity in both cell lines. Cotransduced cells had 2-4 times more alkaline phosphatase activity than would be predicted if effects of individual adenoviruses were additive. We are currently testing whether comparable synergies are seen for bone induction in vivo as well as investigating the mechanistic basis for the observed BMP interactions.

\section{Enhancement of the BMP Responsiveness of Mesenchymal Cells with a Runx2 Adenovirus}

Runx2 is the bone-related product of Cbfa1, a master gene controlling bone and hypertrophic cartilage differentiation [Ducy et al., 2000]. Runx2 is essential for the differentiation of osteoblasts and hypertrophic chondrocytes, and, for this reason, the skeletons of Cbfa 1-/- mice are totally devoid of mineral [Komori et al., 1997; Otto et al., 1997]. Overexpression of Runx 2 in mesenchymal cells can increase osteoblast-specific gene expression at least in part by directly binding to enhancer sequences in target genes [Ducy et al., 1997], although it is likely that some of its actions are indirect and involve induction of other downstream factors. Since BMPs can upregulate Runx2 
in certain systems [Ducy et al., 1997; Lee et al., 2000], it is likely that some of the actions of BMPs may be mediated by this transcription factor. However, other BMP activities are clearly Runx2-independent. For example, in response to BMP treatment and receptor activation, RSmad-Smad4 complexes can directly interact with and activate certain target genes in the absence of Runx2 [Jonk et al., 1998; Hanai et al., 1999]. Consistent with these results, BMP2 can induce the expression of osteocalcin and alkaline phosphatase in cells from $\mathrm{Cbfa} 1-/-$ mice [Komori et al., 1997]. Taken together, these studies suggest that BMPs and Runx2 may stimulate bone formation through distinct, but complimentary pathways and, furthermore, that it may be possible to enhance the osteogenic activity of mesenchymal precursor cells by transduction with a Runx 2 expression vector.

To begin exploring possible interactions between BMPs and Runx2, we conducted studies to determine the extent to which a Runx2 adenovirus can induce functional osteoblast differentiation in the C3H10T1/2 mesenchymal cell line, and then to examine functional interactions between Runx2 and BMP2 in stimulation of osteoblast differentiation in vitro and in vivo [Yang et al., 2003]. AdCMV-Runx2 induced osteoblast markers, such as alkaline phosphatase and osteocalcin, in C3H10T1/2 cells in vitro, and weakly stimulated extracellular matrix mineralization. In contrast, cells transduced with AdCMV-BMP2 exhibited higher levels of mineralization, but expressed barely detectible levels of alkaline phosphatase, Runx2 and osteocalcin. Significantly, when cells were transduced with optimal titers of both AdCMV-Runx2 and AdCMVBMP2, osteoblast differentiation was stimulated to levels that were up to 10 -fold greater than those seen with either AdCMV-Runx2 or AdCMV-BMP2 alone. These results showed that AdCMV-Runx2 transduction increased the sensitivity of cells to AdCMV-BMP2 treatment. To measure in vivo osteogenic activity, virally transduced cells were subcutaneously implanted into immunodeficient mice. As shown in figure 8, cells transduced with control virus only produced fibrous tissue while those with AdCMV-Runx 2 produced limited amounts of both cartilage and bone, as would be predicted from the known effects of Runx 2 in the formation of both bone and cartilage while cells transduced with either AdCMV-BMP2 alone or AdCMV-BMP2 plus AdCMV-Runx2 induced prominent ossicles containing cartilage, bone and a marrow cavity. However, ossification in the AdCMV-BMP2 plus AdCMV-Runx2 group was more extensive. Both mineral content and fractional bone area were greater than that seen with AdCMV-BMP2 alone. The increase in bone area was mainly due to enhanced deposition of cortical bone in the AdCMV-BMP2 plus AdCMV-Runx2 group. These results show that the responsiveness of osteoprogenitor cell populations to BMPs can be enhanced in vitro and in vivo by factors like Runx 2 that are major regulators of the osteoprogenitor lineage. In addition, they suggest possible therapeutic benefits that may be derived from using bone-related transcription factors to enhance BMP responsiveness in osteoprogenitor populations.

\section{Future Directions}

The studies just described highlight the potential for using gene therapy to express unique combinations of regenerative molecules. In addition to the two cases discussed, other unique combinations of factors may enhance bone formation. For example, several FGFs are expressed at early stages of bone development and fracture repair. These molecules control diverse events ranging from the initiation of limb formation and cranial suture mineralization during development to proliferation of bone precursors in the fracture callus [Martin, 1998; Khan et al., 2000; Tickle, 2002]. In particular, FGF2 has potent anabolic effects on bone in vivo and can stimulate bone regeneration when implanted in a suitable matrix [Liang et al., 1999; Pun et al., 2000; Lisignoli et al., 2002]. However, because a major function of FGFs is to stimulate precursor cell proliferation, they can be antagonistic to differentiation factors like BMPs if both factors are simultaneously presented to cells [Terkeltaub et al., 1998]. One possible way to overcome this problem would be to control the temporal sequence of factor expression. For example, a regeneration strategy might be envisioned where early FGF2 expression could be used to expand precursor cells in a fracture callus followed by induction of a BMP to stimulate chondrocyte and osteoblast differentiation. Recently described gene therapy vectors that incorporate inducible promoters may be particularly useful in this regard. For example, Rivera et al. [1999] described a retroviral vector that contains an inducible promoter controlled by rapamycin. Because of its high oral bioavailability, rapamycin can be easily administered in the diet. On being absorbed, it interacts with a twocomponent engineered transcription factor in virally transduced cells to activate the target gene of choice. Using such a system, regenerative strategies can be envisioned in which an early proliferative signal (e.g. FGF2) under the control of an adenovirus is followed by a differ- 
entiation signal (e.g. a BMP) under the control of an inducible promoter. In this way, precursor populations could be expanded before initiation of a differentiation signal. Similar approaches might also be considered for protein therapy in which controlled protein release scaf- folds [Lutolf et al., 2003] are used for the timed release of specific combinations of regenerative molecules. In the future, these and similar approaches may allow control of both the amount and type of bone formed at specific regenerative sites.

\section{References}

Baltzer, A.W., C. Lattermann, J.D. Whalen, P. Wooley, K. Weiss, M. Grimm, S.C. Ghivizzani, P.D. Robbins, C.H. Evans (2000) Genetic enhancement of fracture repair: Healing of an experimental segmental defect by adenoviral transfer of the BMP-2 gene. Gene Ther 7: 734739.

Bergelson, J.M., J.A. Cunningham, G. Droguett, E.A. Kurt-Jones, A. Krithivas, J.S. Hong, M.S. Horwitz, R.L. Crowell, R.W. Finberg (1997) Isolation of a common receptor for Coxsackie $B$ viruses and adenoviruses 2 and 5. Science 275: 1320-1323.

Bonadio, J., E. Smiley, P. Patil, S. Goldstein (1999) Localized, direct plasmid gene delivery in vivo: Prolonged therapy results in reproducible tissue regeneration. Nat Med 5: 753-759.

Ducy, P., T. Schinke, G. Karsenty (2000) The osteoblast: A sophisticated fibroblast under central surveillance. Science 289: 1501-1504.

Ducy, P., R. Zhang, V. Geoffroy, A.L. Ridall, G. Karsenty (1997) Osf2/Cbfa1: A transcriptional activator of osteoblast differentiation. Cell 89 : 747-754.

Franceschi, R.T., D. Wang, P.H. Krebsbach, R.B Rutherford (2000) Gene therapy for bone formation: In vitro and in vivo osteogenic activity of an adenovirus expressing BMP7. J Cell Biochem 78: 476-486.

Hacein-Bey-Abina, S., C. von Kalle, M. Schmidt, F. Le Deist, N. Wulffraat, E. McIntyre, I. Radford, J.L. Villeval, C.C. Fraser, M. CavazzanaCalvo, A. Fischer (2003) A serious adverse event after successful gene therapy for X-linked severe combined immunodeficiency. N Engl J Med 348: 255-256.

Hanai, J., L.F. Chen, T. Kanno, N. Ohtani-Fujita, W.Y. Kim, W.H. Guo, T. Imamura, Y. Ishidou, M. Fukuchi, M.J. Shi, J. Stavnezer, M. Kawabata, K. Miyazono, Y. Ito (1999) Interaction and functional cooperation of PEBP2/ $\mathrm{CBF}$ with Smads. Synergistic induction of the immunoglobulin germline Calpha promoter. J Biol Chem 274: 31577-31582.

Hardy, S., M. Kitamura, T. Harris-Stansil, Y. Dai, M.L. Phipps (1997) Construction of adenovirus vectors through Cre-lox recombination. J Virol 71: 1842-1849.

Hartigan-O'Connor, D., A. Amalfitano, J.S. Chamberlain (1999) Improved production of gutted adenovirus in cells expressing adenovirus preterminal protein and DNA polymerase. J Virol 73: 7835-7841.
Jonk, L.J., S. Itoh, C.H. Heldin, P. ten Dijke, W. Kruijer (1998) Identification and functional characterization of a Smad binding element (SBE) in the JunB promoter that acts as a transforming growth factor-beta, activin, and bone morphogenetic protein-inducible enhancer. J Biol Chem 273: 21145-21152.

Katagiri, T., A. Yamaguchi, M. Komaki, E. Abe, N. Takahashi, T. Ikeda, V. Rosen, J.M. Wozney, A. Fujisawa-Sehara, T. Suda (1994) Bone morphogenetic protein-2 converts the differentiation pathway of $\mathrm{C} 2 \mathrm{C} 12$ myoblasts into the osteoblast lineage. J Cell Biol 127: 1755-1766.

Khan, S.N., M.P. Bostrom, J.M. Lane (2000) Bone growth factors. Orthop Clin North Am 31: 375-388.

Komori, T., H. Yagi, S. Nomura, A. Yamaguchi, K. Sasaki, K. Deguchi, Y. Shimizu, R.T. Bronson, Y.H. Gao, M. Inada, M. Sato, R. Okamoto, Y. Kitamura, S. Yoshiki, T. Kishimoto (1997) Targeted disruption of Cbfal results in a complete lack of bone formation owing to maturational arrest of osteoblasts. Cell 89: 755-764.

Krebsbach, P.H., K. Gu, R.T. Franceschi, R.B. Rutherford (2000) Gene therapy-directed osteogenesis: BMP-7-transduced human fibroblasts form bone in vivo. Hum Gene Ther 11: 1201-1210.

Kwok, K.Y., Y. Yang, K.G. Rice (2001) Evolution of cross-linked non-viral gene delivery systems. Curr Opin Mol Ther 3: 142-146.

Laurencin, C.T., M.A. Attawia, L.Q. Lu, M.D Borden, H.H. Lu, W.J. Gorum, J.R. Lieberman (2001) Poly(lactide-co-glycolide)/hydroxylapatite delivery of BMP-2-producing cells: A regional gene therapy approach to bone regeneration. Biomaterials 22: 1271-1277.

Lee, K.S., H.J. Kim, Q.L. Li, X.Z. Chi, C. Ueta, T. Komori, J.M. Wozney, E.G. Kim, J.Y. Choi, H.M. Ryoo, S.C. Bae (2000) Runx2 is a common target of transforming growth factor beta 1 and bone morphogenetic protein 2, and cooperation between Runx 2 and Smad5 induces osteoblast-specific gene expression in the pluripotent mesenchymal precursor cell line $\mathrm{C} 2 \mathrm{C} 12$. Mol Cell Biol 20: 8783-8792.

Liang, H., S. Pun, T.J. Wronski (1999) Bone anabolic effects of basic fibroblast growth factor in ovariectomized rats. Endocrinology 140 . 5780-5788.
Lieberman, J.R., A. Daluiski, S. Stevenson, L. Wu, P. McAllister, Y.P. Lee, J.M. Kabo, G.A. Finerman, A.J. Berk, O.N. Witte (1999) The effect of regional gene therapy with bone morphogenetic protein-2-producing bone-marrow cells on the repair of segmental femoral defects in rats. J Bone Joint Surg Am 81: 905-917.

Lisignoli, G., M. Fini, G. Giavaresi, A.N. Nicoli, S. Toneguzzi, A. Facchini (2002) Osteogenesis of large segmental radius defects enhanced by basic fibroblast growth factor activated bone marrow stromal cells grown on non-woven hyaluronic acid-based polymer scaffold. Biomaterials 23: 1043-1051.

Lutolf, M.P., F.E. Weber, H.G. Schmoekel, J.C Schense, T. Kohler, R. Muller, J.A. Hubbell (2003) Repair of bone defects using synthetic mimetics of collagenous extracellular matrices. Nat Biotechnol 21: 513-518

Lyons, K.M., B.L. Hogan, E.J. Robertson (1995) Colocalization of BMP 7 and BMP 2 RNAs suggests that these factors cooperatively mediate tissue interactions during murine development. Mech Dev 50: 71-83.

Mahr, J.A., L.R. Gooding (1999) Immune evasion by adenoviruses. Immunol Rev 168: 121-130.

Martin, G.R. (1998) The roles of FGFs in the early development of vertebrate limbs. Genes Dev 12: $1571-1586$

Mason, J.M., D.A. Grande, M. Barcia, R. Grant, R.G. Pergolizzi, A.S. Breitbart (1998) Expression of human bone morphogenic protein 7 in primary rabbit periosteal cells: Potential utility in gene therapy for osteochondral repair. Gene Ther 5: 1098-1104.

Neumann, R., J. Chroboczek, B. Jacrot (1988) Determination of the nucleotide sequence for the penton-base gene of human adenovirus type 5 Gene 69: 153-157.

Nishimatsu, S., G.H. Thomsen (1998) Ventral mesoderm induction and patterning by bone morphogenetic protein heterodimers in Xenopus embryos. Mech Dev 74: 75-88.

Noguchi, P. (2003) Risks and benefits of gene therapy. N Engl J Med 348: 193-194.

Okubo, Y., K. Bessho, K. Fujimura, T. Iizuka, S.I. Miyatake (2000) Osteoinduction by bone morphogenetic protein-2 via adenoviral vector under transient immunosuppression. Biochem Biophys Res Commun 267: 382-387.

Otsuka, E., A. Yamaguchi, S. Hirose, H. Hagiwara (1999) Characterization of osteoblastic differentiation of stromal cell line ST2 that is induced by ascorbic acid. Am J Physiol 277: C132-C138. 
Otto, F., A.P. Thornell, T. Crompton, A. Denzel, K.C. Gilmour, I.R. Rosewell, G.W. Stamp, R.S. Beddington, S. Mundlos, B.R. Olsen, P.B. Selby, M.J. Owen (1997) Cbfa1, a candidate gene for cleidocranial dysplasia syndrome, is essential for osteoblast differentiation and bone development. Cell 89: 765-771.

Pun, S., C.L. Florio, T.J. Wronski (2000) Anabolic effects of basic fibroblast growth factor in the tibial diaphysis of ovariectomized rats. Bone 27: 197-202.

Riew, K.D., J. Lou, N.M. Wright, S.L. Cheng, K.T. Bae, L.V. Avioli (2003) Thoracoscopic intradiscal spine fusion using a minimally invasive gene-therapy technique. J Bone Joint Surg Am 85-A: 866-871.
Rivera, V.M., X. Ye, N.L. Courage, J. Sachar, F. Cerasoli, Jr., J.M. Wilson, M. Gilman (1999) Long-term regulated expression of growth hormone in mice after intramuscular gene transfer. Proc Natl Acad Sci USA 96: 8657-8662.

Rutherford, R.B. (2001) BMP-7 gene transfer to inflamed ferret dental pulps. Eur J Oral Sci 109: 422-424.

Rutherford, R.B., M. Moalli, R.T. Franceschi, D Wang, K. Gu, P.H. Krebsbach (2002) Bone morphogenetic protein-transduced human fibroblasts convert to osteoblasts and form bone in vivo. Tissue Eng 8: 441-452.

Taylor, S.M., P.A. Jones (1979) Multiple new phenotypes induced in 10T1/2 and 3T3 cells treated with 5-azacytidine. Cell 17: 771-779.

Terkeltaub, R.A., K. Johnson, D. Rohnow, R Goomer, D. Burton, L.J. Deftos (1998) Bone morphogenetic proteins and bFGF exert opposing regulatory effects on $\mathrm{PTHrP}$ expression and inorganic pyrophosphate elaboration in immortalized murine endochondral hypertrophic chondrocytes (MCT cells). J Bone Miner Res 13: 931-941.
Tickle, C. (2002) Molecular basis of vertebrate limb patterning. Am J Med Genet 112: 250-255.

Yagi, K., K. Tsuji, A. Nifuji, K. Shinomiya, K. Nakashima, B. DeCrombrugghe, M. Noda (2003) Bone morphogenetic protein-2 enhances osterix gene expression in chondrocytes. J Cell Biochem 88: 1077-1083.

Yang, S., D. Wei, D. Wang, M. Phimphilai, P.H. Krebsbach, R.T. Franceschi (2003) In vitro and in vivo synergistic interactions between the Runx2/Cbfa1 transcription factor and bone morphogenetic protein-2 in stimulating osteoblast differentiation. J Bone Miner Res 18 705-715. 